\title{
Approximately Truthful Mechanisms for Radio Spectrum Allocation
}

\author{
Qinhui Wang, Baoliu Ye, Member, IEEE, Tianyin Xu, \\ Sanglu Lu, Member, IEEE, and Song Guo, Senior Member, IEEE
}

\begin{abstract}
In wireless networks, a recent trend is to make spectrum access dynamic for the sake of efficient utilization of spectrum. In such a scenario, the spectrum is periodically allocated to wireless users using an auction-based market mechanism. A critical property required for designing such mechanism is truthfulness which could avoid market manipulation. Such mechanism design typically involves solving NP-hard problems and hence approximation algorithms are always resorted in real systems. However, recent results suggest that it impossible to implement reasonable approximations without losing robustness to manipulation. In this paper, we solve the problem in a novel perspective by relaxing the constraints of ensuring strong truthfulness. We discuss the concepts of approximate truthfulness and provide approximately truthful mechanisms to improve efficiency (in terms of social welfare and spectrum utilization). We first develop a computationally efficient mechanism that achieves truthful in expectation. This mechanism is based on the assumption that bidders are risk-neutral. Following that, we break the assumption by proposing a hard-to-manipulate auction, which makes it hard to manipulate the auction for profit gains. Our extensive simulation results show that our mechanisms can achieve significant improvement over the state-of-the-art mechanisms.
\end{abstract}

Index Terms-Dynamic Spectrum Access, Spectrum Auctions, Approximate Truthfulness, Algorithms.

\section{INTRODUCTION}

$\mathbf{R}$ ADIO spectrum is a critical but scarce resource for wireless communications. Usage of spectrum has long been governed by government agencies (e.g., FCC in USA) who allocate spectrum by assigning licenses. Only licensed users are allowed to use the spectrum. Such kind of spectrum allocation policy is in a very long term fashion with space-time invariance. Recently, with the fast development of wireless devices and applications, it has been widely recognized that the spectrum is becoming increasingly crowded under the longterm and exclusive management policy. However, It has been widely understood that most of the licensed spectrum is underutilized [1], [2].

With the advances in cognitive radio techniques, Dynamic Spectrum Access (DSA) has been proposed to address the

Copyright (c) 2013 IEEE. Personal use of this material is permitted. However, permission to use this material for any other purposes must be obtained from the IEEE by sending a request to pubs-permissions@ieee.org.

Q. Wang, B. Ye and S. Lu are with National Key Laboratory for Novel Software Technology, Nanjing University, P.R. China (E-mail: qhwang@dislab.nju.edu.cn, \{yebl, sanglu\}@nju.edu.cn). Q. Wang is also with Department of Management, Army Command College, P.R. China. T. Xu is with Department of Computer Science and Engineering, University of California, San Diego, USA (E-mail: xuty1985@gmail.com). S. Guo is with School of Computer Science and Engineering, University of Aizu, Japan (Email: sguo@u-aizu.ac.jp). above dilemma [3], [4]. Under DSA, licensed users (called primary users) are encouraged to open up their idle spectrum to unlicensed users (called secondary users). It is a win-win situation because that primary users can obtain financial gains by leasing their idle spectrum and the service requirements of secondary users can be satisfied. Auctions are widely accepted as an efficient approach to re-distribute spectrum among users due to the perceived fairness and allocation efficiency [5]. Recently, designing auctions for re-distributing the spectrum among secondary users (called Secondary Spectrum Auction, SSA) has attracted lots of research interest [11]-[16].

A typical SSA is made up of a lot of small secondary users distributed over a relatively small geographic region. As a result, an efficient SSA is required to be quickly conducted. In addition, spectrum reusability can be exploited to improve spectrum utilization, which means that the same spectrum band can be allocated to multiple well-separated bidders. Spectrum reusability constitutes the fundamental difference between spectrum auctions and conventional goods auctions (e.g., painting auctions). A critical property required for designing auction, including spectrum auction, is truthfulness (i.e., strategy-proofness). Truthful auction could avoid market manipulation which is a deliberate attempt to interfere with the free and fair operation of the market and create artificial, false or misleading appearances with respect to the price of market. Truthfulness makes life easier for bidders. Otherwise, each bidder has to figure out the others' bidding strategies before being able to get an optimal bidding strategy for herself.

One of the most attractive solutions to the above problem is the VCG mechanism [17]-[19] which has advantages of being truthful and allocatively efficient (it maximizes the value of the allocation over all bidders) [33]. However, such solution has the following problems. First, exploiting spectrum reusability leads to addressing the interference constraints, which make the problem of finding optimal spectrum allocation be NPHard [7]. Therefore, approximate algorithms are always resorted in practical. However, approximate algorithms make ensuring truthfulness via VCG is impossible [22]. This is because the VCG mechanism fails to be truthful when one does not have access to optimal solution [9], [10]. On another hand, traditional truthful auctions, e.g., k-position [24], will lose truthfulness or become computationally prohibitive when applied to spectrum auctions due to the spatial reuse of spectrum, i.e., a spectrum band can be assigned to multiple well-separated bidders [7], [14].

As the problem of finding the optimal spectrum allocation under interference constraints is NP-hard, we introduce the 
concept of approximate truthfulness to release the burden of providing strong truthfulness. Approximate truthfulness is sufficient to guarantee bidders not to lie in auctions [22], [25]. Moreover, it is helpful to make the mechanism designer focus on designing efficient allocation algorithm to improve allocative efficiency (including social welfare and spectrum utilization) [8]. We first propose a computationally efficient mechanism that achieves truthful in expectation. It guarantees any bidder cannot gain positive profit in expectation from non-truthful bid. In this situation, bidders are assumed to be risk-neutral, i.e., bidders will have no preference between risk aversion and risk seeking. Following that, we attempt to break this assumption by relaxing truthfulness to a type of hardness of manipulation, which guarantees that it is NP-hard to gain at least any non-negative profit from non-truthful bid for any bidder. In this situation, we move the difficulty of manipulation from the mechanism to the manipulators, thereby achieving a reasonable and tractable mechanism.

In this paper, we first provide a near optimal algorithm via linear programming. The algorithm is shown to be monotone, and thus a novel pricing scheme is proposed to ensure the mechanism achieving truthful in expectation. Following that, we attempt local improvements on the monotone algorithm which improves the economic efficiency but unravels the monotonicity. Therefore, the previous pricing scheme is no longer suitable. In this case, we show that such improved algorithm combined with a VCG-based payment scheme is hard-to-manipulate.

The concept of approximate truthfulness is firstly introduced in our conference paper [8] for spectrum auction design, where an auction achieving truthful in expectation for strict request model (named as ETEX) is proposed. ETEX is based on the assumption that bidders are risk-neutral. This paper extends it mainly by proposing an additional approximately truthful mechanism which breaks the above assumption and improves efficiency.

The main contributions of our work in this paper can be summarized as follows:

1) To the best of our knowledge, we are the first to introduce the concept of approximate truthfulness, which can release the burden of providing strong truthfulness to improve the allocation efficiency, for spectrum allocation in DSA.

2) We provide a near-optimal algorithm, and we combine it with a novel pricing method to ensure that the mechanism achieves truthful in expectation in polynomial complexity.

3) We attempt local improvements based on the monotone algorithm to improve allocation efficiency. Although the previous pricing method is unsuitable, we show such an efficient algorithm combined with a VCG-payment scheme is hard-to-manipulate.

4) Through extensive simulations, we show that our mechanisms outperform existing truthful mechanisms by about $23 \% / 27 \%$ in average in terms of social welfare and spectrum utilization.

The rest of the paper is organized as follows. Sec. II reviews the related work. Sec. III introduces the preliminaries on auctions. The network model and problem statement are given in Sec. IV. The auction achieving truthful in expectation is proposed in Sec. V. We analyze the properties of ETEX in Sec. VI. The hard-to-manipulate auction is described in Sec. VII. Experimental results are shown in Sec. VIII. Sec. IX concludes the paper.

\section{RELATED WORK}

How to efficiently allocate spectrum while enabling spectrum reuse has attracted great research interests. Gandhi et al. [12] propose a real-time spectrum auctions framework which considers interference constraints. To obtain computationally efficient mechanisms, they choose to linearize the interference constraints. Subramanian et al. [13] propose a greedy graphcoloring based algorithm to approach the optimal revenue. However, these auctions do not consider truthfulness.

Zhou et al. [14] firstly investigate the design of truthful SSA and show that a greedy algorithm coupled with a VCGpayment scheme will lose truthfulness. They propose VERITAS [14] which consists of a greedy allocation algorithm and a critical value based pricing scheme. Recently, this work has been extended to consider double auctions [16]. In [21], the authors aim to find a trade-off between social welfare and fairness maintained. The other two works [15], [20] attempt to maximize the expected revenue for cellular networks under Bayesian settings, wherein each bidder's valuation value is drawn from a known probability distribution. In another work, Wu et al. [32] design a spectrum auction mechanism based on the VCG mechanism. However, their altered payment scheme destroys the truthfulness property. Moreover, their mechanism requires solving an integer linear programming (NP-hard) problem, which makes their approach impractical for large scales networks.

As another line of related works, there has been considerable interest in recent years in characterizing approximately truthful combinatorial auctions in economics, since the combinatorial allocation problem is both NP-complete [28] and inapproximable [29]. Feigenbaum et al. [30] have defined the concept of strategically faithful approximations and proposed the study of approximation as an important direction for algorithmic mechanism design. Sanghvi et al. [25] force a bidder to reveal information by making it too hard for that same bidder to avoid revelation, i.e., any attempt to backsolve the computation (to manipulate the result) will meet with difficulty. Archer et al. [22] adopt similar notions of approximate truthfulness on single-minded combinatorial auctions and propose a computationally efficient auction that achieves both truthful with error probability and truthful in expectation.

Inspired by these work, we apply the approximate truthfulness in spectrum auction design to achieve a tradeoff between allocation efficiency and truthfulness.

\section{PRELIMINARIES ON AUCTION}

An auction $M(A, P)$ consists of an allocation algorithm $A$ along with a pricing scheme $P$. All bidders submit the bids and requests to the auctioneer simultaneously. After collecting bid vector $\mathbf{b}=\left(b_{1}, b_{2}, \cdots, b_{N}\right)$ and demand vector $\mathbf{d}=$ 
$\left(d_{1}, d_{2}, \cdots, d_{N}\right)$ from all bidders, the auctioneer determines the winning bidders and allocates goods to them according to the allocation algorithm $A$. A price $p_{i}$ will be charged from each winner $i$ according to the pricing scheme $P$. For its requested goods, each bidder $i$ has a true valuation $v_{i}$, which is private for other bidders. The utility (i.e., profit) of each bidder $i$ is computed as:

$$
u_{i}\left(b_{i}, \mathbf{b}_{-i}\right)= \begin{cases}v_{i}-p_{i}, & \text { if } i \text { wins } \\ 0, & \text { otherwise }\end{cases}
$$

where $\mathbf{b}_{-i}$ represents the bidders' bids except bidder $i$, i.e., $\mathbf{b}_{-i}=\left(b_{1}, \cdots, b_{i-1}, b_{i+1}, \cdots, b_{N}\right)$.

A common goal in the auction design is to maximize the social welfare, which is defined as the sum of bids submitted by all winners. In the following, we summarize the basic concepts and properties of truthful and approximately truthful auction mechanisms.

\section{A. Truthful Auction Mechanisms}

Since bidders are selfish, a bidder may choose to submit a bid $b_{i}\left(b_{i} \neq v_{i}\right)$ to maximize its utility. An auction is truthful if for any bidder $i$, the utility $u_{i}$ is maximized when biding the true value $v_{i}$, that is $u_{i}\left(v_{i}, \mathbf{b}_{-i}\right)>u_{i}\left(b_{i}, \mathbf{b}_{-i}\right)$ for any bid $b_{i}$ when fixing $\mathbf{b}_{-i}$. Truthful auctions will guarantee that no bidders can obtain additional profit gains by lying on bids.

The Vickrey-Clarke-Groves (VCG) mechanism [17]-[19] is famous for securing truthful bids from bidders. A mechanism $M(A, P)$ belongs to the class of VCG mechanism if and only if the allocation algorithm $A$ finds the optimal allocation that maximizes the social welfare and the price charged from each bidder $i$ is:

$$
p_{i}=W_{A}\left(\mathbf{b}_{-i}\right)-\left(W_{A}(\mathbf{b})-b_{i}\right),
$$

where $W_{A}\left(\mathbf{b}_{-i}\right)$ and $W_{A}(\mathbf{b})$ denote the optimal social welfare returned by running $A$ with all bidders and all other bidders except $i$, respectively. Note that the VCG mechanism fails to be truthful when $A$ does not get the optimal allocation [9], [10].

\section{B. Approximately Truthful Auction Mechanisms}

Recently, the approximate truthfulness has received considerable interest for combinatorial auction design in economics. In this paper, we introduce this concept to spectrum auction design. We consider two types of approximately truthful auctions: truthful-in-expectation and hard-to-manipulation, as defined below.

Definition 1 (Truthful-in-Expectation): A randomized mechanism is truthful-in-expectation if for each bidder $i$, the equation $E\left[u_{i}\left(v_{i}, \mathbf{b}_{-i}\right)\right] \geq E\left[u_{i}\left(b_{i}, \mathbf{b}_{-i}\right)\right]$ holds for any bid $b_{i}$ when fixing $\mathbf{b}_{-i}$.

Definition 2 (Hard-to-Manipulate): A mechanism is hardto-manipulate if it is NP-hard for any bidder $i$ to gain at least any non-negative additional profit by lying on bids.

The truthful-in-expectation mechanism let lying bidders take risks of losing profit. Therefore, a bidder under such a mechanism is generally assumed to be risk-neutral, i.e., a bidder is reluctant to bear the risk via lying. The hardto-manipulate mechanism breaks the assumption by making it hard to manipulate the auction for profit gains. It moves the difficulty of the manipulation from the mechanism to the manipulators, which makes it hard for profit gain via lying.

\section{Network Model and Problem Statement}

\section{A. Network Model}

In generally, we refer the primary user and its associated secondary users as auctioneer and bidders, respectively. The set of bidders are denoted as $\mathcal{N}(|\mathcal{N}|=N)$. The spectrum to be auctioned is divided into $K$ identical channels denoted as $\mathcal{K}(|\mathcal{K}|=K)$. Each bidder submits its bid $b_{i}\left(b_{i}>0\right)$ to the auctioneer to request $d_{i}\left(0<d_{i} \leq K\right)$ channels. Strict request model is adopted here, where each bidder will win either all $d_{i}$ channels or nothing. We consider sealed-bid auctions where all bidders simultaneously submit their bids to the auctioneer and we assume that the bidders do no collude.

In this paper, we focus on the widely-used protocol interference model [6], [7], a succinct model to formulate the impact of interference within resource allocation problems, in order to highlight our contributions in auction mechanisms. With the protocol model employed, the interference can be well captured by a conflict graph $G(\mathcal{N}, \mathcal{E})$, where $\mathcal{E}$ is the collection of all edges [7]. An edge $(i, j)$ belongs to $\mathcal{E}$ if bidders $i$ and $j$ conflict with each other when they use the same channel simultaneously. Let $\mathbb{N}(i)$ be the set of bidders that interfere with $i$ (i.e., the neighboring nodes of $i$ in $G$ ).

\section{B. Problem Formulation}

In this paper, we focus on maximizing social welfare which is a strong indicator of how efficiently the buyers make use of the sold spectrum bands. We use the following binary variables $x_{i}$ and $a_{i k}$ to formally describe the allocation problem.

$$
\begin{aligned}
x_{i} & = \begin{cases}1, & \text { if bidder } i \text { is a winner, } \\
0, & \text { otherwise. }\end{cases} \\
a_{i k} & = \begin{cases}1, & \text { if channel } k \text { is assigned to bidder } i, \\
0, & \text { otherwise }\end{cases}
\end{aligned}
$$

The channel allocation to achieve maximum social welfare can thus be formulated as an Integer Programming (IP) problem:

$$
\begin{array}{r}
\max \sum_{i=1}^{N} b_{i} x_{i} \quad \text { s.t. } \\
\sum_{k=1}^{K} a_{i k}=x_{i} d_{i}, \quad \forall i \in \mathcal{N}, \\
a_{i k}+a_{j k} \leq 1, \quad \forall(i, j) \in \mathcal{E}, \forall k \in \mathcal{K}, \\
x_{i}, a_{i k} \in\{0,1\}, \quad \forall i \in \mathcal{N}, \forall k \in \mathcal{K} .
\end{array}
$$

Constraints in (6) ensure that the request is strict, i.e., a bidder obtains either $d_{i}$ or 0 channels. The interference constraints require that any two bidders $i$ and $j$ sharing an edge are not allowed to be assigned to the same channel, i.e., $\forall k \in \mathcal{K}, a_{i k} a_{j k}=0$ if $\forall(i, j) \in \mathcal{E}$, which can be linearized 
by (7) due to the integer constraints in (8). This optimization problem is named as SA-SR (Spectrum Allocation with Strict Request). The following theorem shows it is NP-hard and inapproximable.

Theorem 1: The SA-SR problem is NP-hard. Moreover, it does not admit any $\delta$-approximation algorithm unless $P=N P$, where $\delta$ is some positive constant.

Proof: Consider a special case with $d_{i}=K=1, \forall i \in \mathcal{N}$, the channel will be allocated to an independent set in conflict graph (addressing interference constraints) with maximized summation of bidders' valuation. Therefore, finding the optimal social welfare for this case is equivalent to finding the maximum-weight independent set, where the weight of a node is the bid of a bidder. The maximum-weight independent set problem is known to be NP-hard and it is also NP-hard to find a constant-factor approximate algorithm for this problem. The same results hold for the general SA-SR problem as well.

\section{TRUThFul IN EXPECTATION}

In this section, we develop ETEX, an auction achieving truthful in expectation consisting of a suboptimal allocation algorithm (referred as ETEX-Allocation) and a novel pricing scheme (referred as ETEX-Prcing). Here, we assume bidders are risk-neutral, i.e., bidders have no preference between risk aversion and risk seeking.

\section{A. ETEX-Allocation Algorithm}

The allocation algorithm starts by solving linear programming (LP), and it finds a solution based on the LP solutions. The idea is similar with the solution of [31] which approximates a routing problem where band is allocated to nodes according to the LP solution, which is close to be optimal. The ETEX-Allocation is based on the following strategy. First, it runs the linear-programming (LP) relaxation of the problem. Second, it uses the LP results to rank the bidders. Finally, it sequentially allocates the channels to bidders based on the ranking order while satisfying interference constants.

The LP formulation for the problem (5) is simply substituting Equ. (8) by the following equation (see Equ. 9), and the other constraints stays unchanged.

$$
x_{i}, a_{i k} \in[0,1], \quad(\forall i \in \mathcal{N}, \forall k \in \mathcal{K})
$$

Let $B=\left\{x_{1}^{*}, \cdots, x_{n}^{*}\right\}$, where $x_{i}^{*}$ is the LP solution of Equ. (5) for bidder $i$. We use $B^{\prime}=\operatorname{Sort}(B)$ to sort the set $B$ in a descending order. We first use Breadth-First-Search procedure $B F S(G)$ to sort the topology $G(\mathcal{N}, \mathcal{E})$, which ensures the updating process is executed sequentially. We then sequentially allocate channels to bidders from high to low according to the ordered solution set $B^{\prime}$. The procedure $\operatorname{Top}\left(B^{\prime}\right)$ gets the first bidder in $B^{\prime}$. For each bidder $i$, if $|A \operatorname{vai}(i)|$, the number of available channels of bidder $i$, is greater than the request $d_{i}$, we then use $\operatorname{Assign}\left(i, d_{i}\right)$ to assign $d_{i}$ channels from $\operatorname{Avai}(i)$ to $i$. After allocating, we use the updating process $U p d a t e(j)$ to remove the allocated channels from the available channel sets of bidder $j$ for each $j \in \mathbb{N}(i)$. This process ensures that the interference constraints are satisfied. We describe the detailed algorithm in Algorithm 1. In the following section, we will

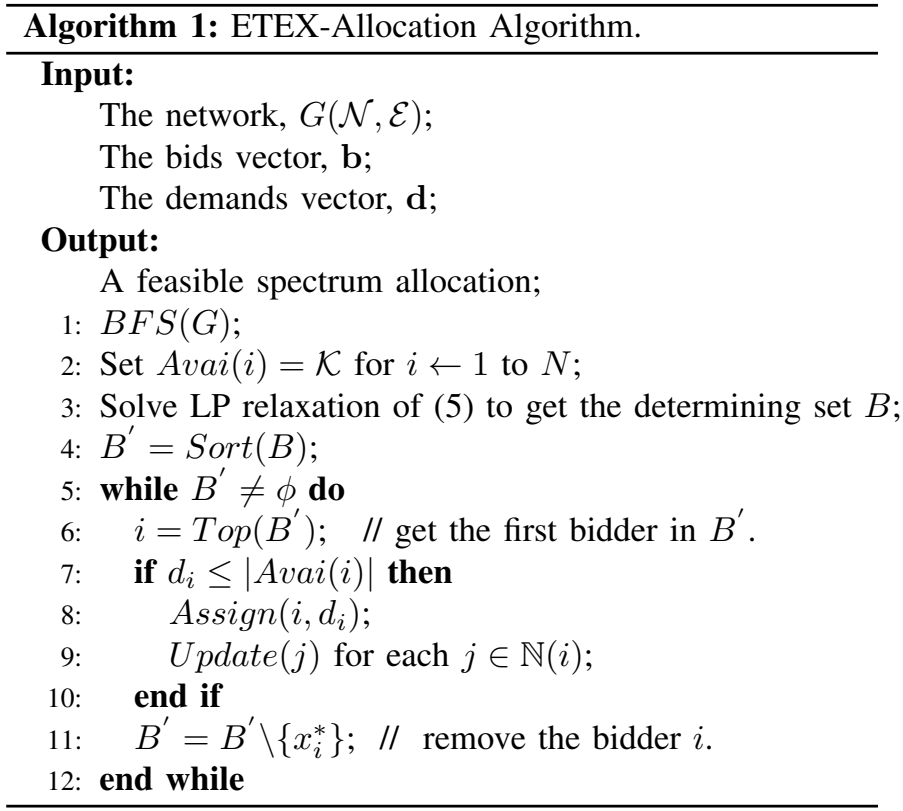

show the ETEX-Allocation is monotone, which means that bidder $i$ will win by bidding higher than $b_{i}$ if it wins by bidding $b_{i}$.

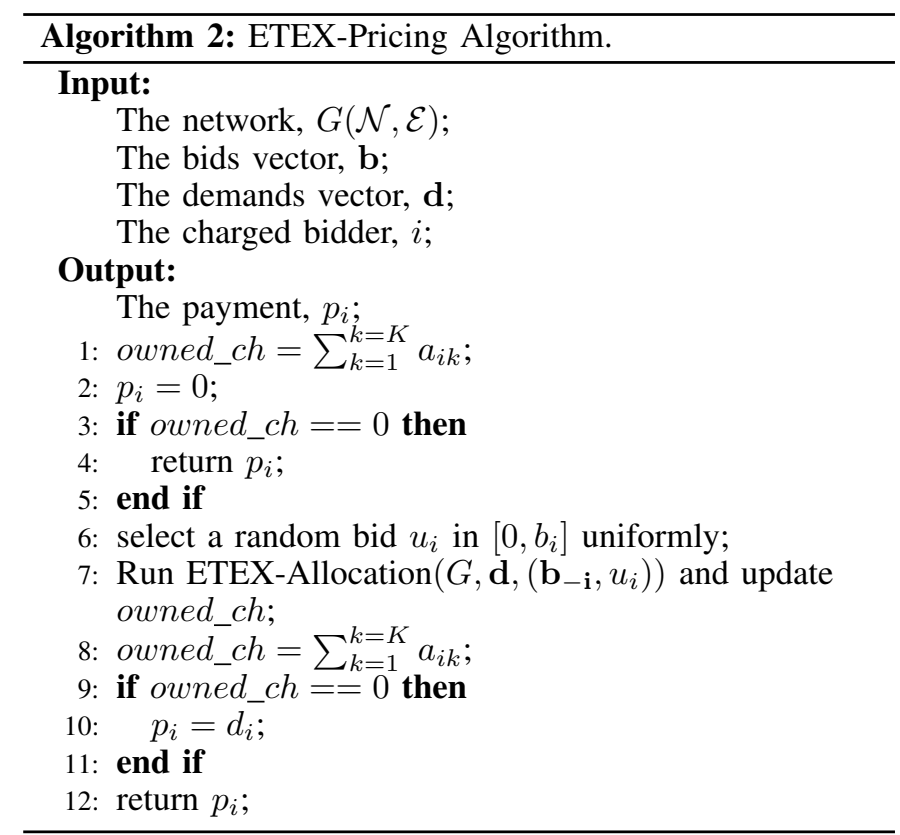

\section{B. ETEX-Pricing Scheme}

A mechanism is truthful if and only if the allocation algorithm is monotone and the pricing method guarantees the payment for any bidder $i$ is its critical value [27]. The monotonicity property ensures that if any bidder $i$ wins by bidding $b_{i}$, it will also win by bidding higher than $b_{i}$. A monotone allocation algorithm implies there is a critical value such that any bidder will win by bidding higher than this value, and will lose by bidding lower than that. Although, ETEX-Allocation is monotone (see Lemma 3), a natural way 
of finding the single critical value requires binary search on the bid $b_{i}$ which requires multiple times of running LP. Instead of providing exact critical value, in this paper, we develop a technique to find the payment approximately to achieve truthful in expectation. This greatly minimizes the computational overhead. The basic idea behind ETEX-Pricing scheme is finding the expected value of critical value for each winner.

In the pricing algorithm, we randomize the price for each winner $i$. Let random variable $p_{i}$ denote the randomized payment. The strategy for determining the value of $p_{i}$ is as follows: select a random bid $u_{i} \in\left[0, b_{i}\right]$ uniformly, and run the ETEX-Allocation once again with bidder $i$ bidding $u_{i}$ (the other bidders' bids do not change). If the bidder $i$ loses, then we set the payment $p_{i}=b_{i}$. Otherwise, i.e., bidder $i$ still wins, we set $p_{i}=0$. The detailed algorithm is described in Algorithm 2, where owned_ch is the number of channels currently owned by $i$. In the following section, we will show that the expected value of the payment from bidder $i$ is its critical value (see Lemma 4).

\section{Properties Analysis}

In this paper, we evaluate the properties of mechanisms in terms of truthfulness and computational complexity. We first prove ETEX is truthful in expectation by showing the expected utility of each bidder $i$ is maximized with truthful bidding, and then we analyze the computational complexity of ETEX.

\section{A. Truthfulness}

To prove ETEX is truthful in expectation, we try to prove ETEX-Allocation is monotone and ETEX-Pricing charges each bidder its expected critical value.

We firstly characterize the properties of ETEX-Allocation algorithm. We start by establishing the following two lemmas.

Lemma 1: $\forall \mathbf{b}_{-i}$, if $b_{i}^{\prime}>b_{i}$, then $x_{i}^{*}\left(b_{i}^{\prime}, \mathbf{b}_{-i}\right) \geq$ $x_{i}^{*}\left(b_{i}, \mathbf{b}_{-i}\right)$, where $x_{i}^{*}\left(b_{i}^{\prime}, \mathbf{b}_{-i}\right)$ and $x_{i}^{*}\left(b_{i}, \mathbf{b}_{-i}\right)$ represent the $L P$ solution of bidder $i$ with bids $\left(b_{i}^{\prime}, \mathbf{b}_{-i}\right)$ and $\left(b_{i}, \mathbf{b}_{-i}\right)$, respectively.

Proof: To simplify the description, we use $\mathbf{S}$ and $\mathbf{S}^{\prime}$ to denote the LP solution vector when bidding $\left(b_{i}, \mathbf{b}_{-i}\right)$ and $\left(b_{i}^{\prime}, \mathbf{b}_{-i}\right)$ respectively (i.e., $S_{i}=x_{i}^{*}\left(b_{i}, \mathbf{b}_{-i}\right)$ and $S_{i}^{\prime}=$ $\left.x_{i}^{*}\left(b_{i}^{\prime}, \mathbf{b}_{-i}\right)\right)$. Since $\mathbf{S}$ is the optimal solution to the linear program, and $\mathbf{S}^{\prime}$ is a feasible solution, we have

$$
\sum_{i=1}^{n} S_{i}^{\prime} b_{i} \leq \sum_{i=1}^{n} S_{i} b_{i}
$$

Similarly, we get

$$
S_{i} b_{i}^{\prime}+\sum_{j \neq i} S_{j} b_{j}^{\prime} \leq S_{i}^{\prime} b_{i}^{\prime}+\sum_{j \neq i} S_{j}^{\prime} b_{j}^{\prime}
$$

Let (11) plus (10), and move the right part of (10) to the left, move the left part of (11) to the right, we get

$$
\left(b_{i}^{\prime}-b_{i}\right)\left(S_{i}^{\prime}-S_{i}\right) \geq 0
$$

As $b_{i}^{\prime}-b_{i}>0$, then $S_{i}^{\prime}-S_{i} \geq 0$, i.e., $x_{i}^{*}\left(b_{i}^{\prime}, \mathbf{b}_{-i}\right) \geq$ $x_{i}^{*}\left(b_{i}, \mathbf{b}_{-i}\right)$.
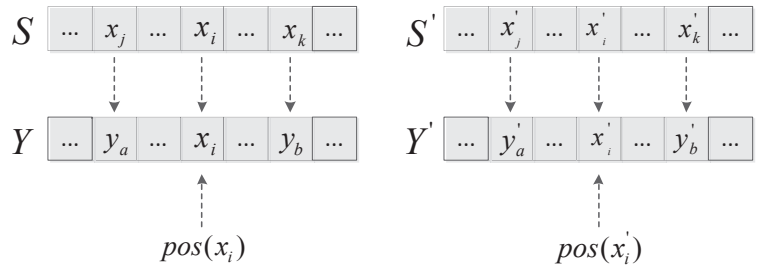

Fig. 1: Two lists of LP solutions ( $\mathbf{S}$ and $\left.\mathbf{S}^{\prime}\right)$ where only bidder $i$ 's bid is different. The lists $\mathbf{Y}$ and $\mathbf{Y}^{\prime}$ are the corresponding LP solutions of $i$ 's neighbors, which are sorted in descending order. $\operatorname{pos}\left(x_{i}\right)$ and $\operatorname{pos}\left(x_{i}^{\prime}\right)$ denote the position in the list of $\mathbf{Y}$ and $\mathbf{Y}^{\prime}$ respectively.

Lemma 2: Consider two bidding lists $\left(\mathbf{b}_{-\mathbf{i}}, b_{i}\right)$ and $\left(\mathbf{b}_{-\mathbf{i}}, b_{i}^{\prime}\right)$, and the corresponding LP solution lists are $\left(\mathbf{x}_{-\mathbf{i}}, x_{i}\right)$ and $\left(\mathbf{x}_{-\mathbf{i}}^{\prime}, x_{i}^{\prime}\right)$, respectively. If $b_{i}<b_{i}^{\prime}$, we have $x_{k} \geq x_{k}^{\prime}$, $\forall k \in \mathbb{N}(i)$, where $\mathbb{N}(i)$ is the set of neighboring bidders of $i$.

Proof: With Lemma 1, we get $x_{i}^{\prime} \geq x_{i}$, which means bidder $i$ will get more fractional spectrum when its bid increases, and the increased spectrum allocated to bidder $i$ is $\Delta d=\left(x_{i}^{\prime}-x_{i}\right) d_{i}$. Since $\left(\mathbf{x}_{-\mathbf{i}}, x_{i}\right)$ and $\left(\mathbf{x}_{-\mathbf{i}}^{\prime}, x_{i}^{\prime}\right)$ are both optimal solutions, bidder $i$ 's increased spectrum would result in decreasing the spectrum of its neighbors. Bidder $i$ increases $\Delta d$ and thus one of its neighbor would reduce at most $\Delta d$ spectrum according to the interference constraints, which means that other bidders can improve at most $\Delta d$ when $b_{i}$ increases. We now assume that there exists one neighbor $k$ of bidder $i$ improves its LP solution, i.e., $x_{k}^{\prime}>x_{k}$. Since $k$ can improve at most $\Delta d$ spectrum yet $k$ 's neighbor $i$ have improved $\Delta d$ spectrum, which means that $k$ cannot improve spectrum anymore by the interference constraints. That is if $x_{k}^{\prime}>x_{k},\left(x_{k}^{\prime}, \mathbf{x}_{-\mathbf{i}}\right)$ would be the optimal solution for $\left(b_{i}, \mathbf{b}_{-\mathbf{i}}\right)$. This is a contradiction which leads to $x_{k}^{\prime} \leq x_{k}, \forall k \in$ $\mathbb{N}(i)$.

With Lemma 1 and Lemma 2, we can prove the ETEXAllocation is monotone.

Lemma 3: The ETEX-Allocation algorithm is monotone, i.e., if bidder $i$ wins by bidding $b_{i}, i$ will also win by bidding $b_{i}^{\prime}$ where $b_{i}^{\prime}>b_{i}$.

Proof: Consider two bidding lists where only bidder $i$ 's bid is different and the corresponding LP solutions are $\mathbf{S}$ and $\mathbf{S}^{\prime}$ respectively (see Fig. 1). In $\mathbf{S}$, the bidder $i$ is allocated by bidding $b_{i}$, and the corresponding LP solution is $x_{i}$. In $\mathbf{S}^{\prime}$, the bidder $i$ bids $b_{i}^{\prime}$ with $b_{i}^{\prime}>b_{i}$, and the corresponding LP solution is $x_{i}^{\prime}$. Let $y_{k}, k \in \mathbb{N}(i)$, be the corresponding LP solutions of the bidder $i$ 's neighbors in $\mathbf{S}$. Let $\mathbf{Y}$ denote the sorted set (in descending order) of $\left\{y_{k}, k \in \mathbb{N}(i)\right\} \cup x_{i}$, and the position of bidder $i$ in $\mathbf{Y}$ is $\operatorname{pos}\left(x_{i}\right)$. Similarly, let $y_{k}^{\prime}, k \in \mathbb{N}(i)$ be the corresponding LP solutions of bidder $i$ 's neighbors in $\mathbf{S}^{\prime}$, and let $\mathbf{Y}^{\prime}$ denote the sorted set of $\left\{y_{k}^{\prime}, k \in\right.$ $\mathbb{N}(i)\} \cup x_{i}^{\prime}$. The position of bidder $i$ in $\mathbf{Y}^{\prime}$ is $\operatorname{pos}\left(x_{i}^{\prime}\right)$. We have $y_{k}^{\prime} \leq y_{k}, k \in \mathbb{N}(i)$ by Lemma 2 . Therefore we get $\operatorname{pos}\left(x_{i}\right) \geq$ $\operatorname{pos}\left(x_{i}^{\prime}\right)$. We know that only bidder $i$ 's neighbors would cause conflicts such that bidder $i$ would not be allocated. Now we prove our claim by contradiction. We assume bidder $i$ loses 


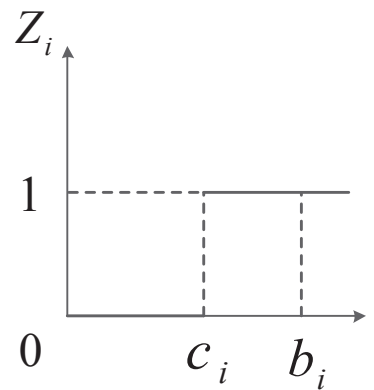

Fig. 2: The graph shows the each bidder $i^{\prime}$ s winning probability $Z_{i}$ as a function of different bids $b_{i}$ when fixing $\mathbf{b}_{-i}$, where $c_{i}$ is critical value.

by bidding $b_{i}^{\prime}$, which means that a neighboring bidder with smaller position has caused conflict to bidder $i$ in $\mathbf{Y}^{\prime}$. Since $\operatorname{pos}\left(x_{i}\right) \geq \operatorname{pos}\left(x_{i}^{\prime}\right)$, the conflicting neighbor will also make bidder $i$ not be allocated in $\mathbf{Y}$ according to allocation rule. This leads to a contradiction.

Now we characterize the properties of ETEX-Pricing algorithm by showing that the charged price for each bidder $i$ is the expected value of its critical value.

Lemma 4: Let $p_{i}$ be the random variable to denote payment charged from bidder $i$, the expected value of $p_{i}$ is its critical value $c_{i}$, i.e., $E\left[p_{i}\right]=c_{i}$.

Proof: We use $Z_{i}$ to denote the overall probability that $i$ wins his desired channels, and $c_{i}$ to denote the critical value. According to the definition of critical value, we know bidder $i$ will be allocated with the requested channels when bidding $b_{i}>c_{i}$, where $c_{i}$ is its critical value. Otherwise, $i$ will lose by bidding lower than $c_{i}$. Therefore, we can describe $Z_{i}\left(b_{i}, \mathbf{b}_{-i}\right)$ as a step function of $b_{i}$ when fixing $\mathbf{b}_{-i}$, as show in Figure 2. Note that $\mathbf{b}_{-i}$ is fixed throughout this discussion, so we suppress it in the notation for convenient. After that, according to the pricing strategy, it is easy to compute the expectation of the payment $p_{i}$ for winner $i$ is $c_{i}$ according the following equation.

$$
E\left[p_{i}\right]=P r_{l o s e} \cdot b_{i}+P r_{w i n} \cdot 0=\frac{c_{i}}{b_{i}} \cdot b_{i}+\left(1-\frac{c_{i}}{b_{i}}\right) \cdot 0=c_{i} .
$$

where $P r_{\text {lose }}$ and $P r_{w i n}$ denote the probability of winning and losing, respectively.

Based on the characterizations of allocation method and pricing scheme, we now show that ETEX is truthful in expectation. With the ETEX-Allocation rule and the pricing scheme, we obtain:

Theorem 2: The ETEX is truthful in expectation.

Proof: Let $v_{i}$ be the truthful bidding of bidder $i$, and $b_{i}$ be the bidding such that $b_{i} \neq v_{i}$. We have the following two cases according to whether bidder $i$ loses or wins:

- CASE 1: $i$ loses by bidding $b_{i}$, then $i$ will also lose by bidding $v_{i}$ by Lemma 3 . Therefore the utility of bidder $i$ is $u_{i}\left(v_{i}, \mathbf{b}_{-i}\right)=u_{i}\left(b_{i}, \mathbf{b}_{-i}\right)=0$.

- CASE 2: $i$ wins by bidding $b_{i}$. If $i$ also wins by bidding $v_{i}$, because the payment of critical value is same, then $E\left[u_{i}\left(b_{i}, \mathbf{b}_{-i}\right)\right]=v_{i}-c_{i}=E\left[u_{i}\left(v_{i}, \mathbf{b}_{-i}\right)\right]$. If $i$ loses by bidding $v_{i}$, then the critical value $c_{i}$ must be greater than $v_{i}$. Therefore the utility by bidding $b_{i}$ is $E\left[u_{i}\left(b_{i}, \mathbf{b}_{-i}\right)\right]=$ $v_{i}-c<0=E\left[u_{i}\left(v_{i}, \mathbf{b}_{-i}\right)\right]$.

In both cases, we have $E\left[u_{i}\left(b_{i}, \mathbf{b}_{-i}\right)\right] \leq E\left[u_{i}\left(v_{i}, \mathbf{b}_{-i}\right)\right]$, this completes the proof.

Computational Complexity: We now analyze the computational complexity of ETEX. Despite the process of LP, the ETEX-Allocation takes $O(N+L)$ to sort the topology, where $L$ is number of edges in $G\left(L \leq \frac{N(N-1)}{2}\right)$, and takes $O(N \log N)$ time to sort $B$. For each process of updating the available channels of the neighbors of $i$, it takes at most $O(N)$ time. That is, ETEX-Allocation runs in $O\left(L+N \log N+N^{2}\right)$. For pricing algorithm, it runs the allocation algorithm again without BFS procedure for each winner. Therefore, the ETEX runs in time $O\left(L+N^{2} \log N+N^{3}\right)$ plus $\sharp+1$ times of the time consumed by the LP process with bounded $K \times N$ parameters [23], where $\sharp$ is the number of winners in the allocation.

Note that we use Karmarkars Algorithm to obtain the LP solution in this paper. Karmarkars Algorithm belongs to the interior point method, which generates a sequence of points inside the feasible region and finally approaches the optimal vertex [23] and its computational complexity is bounded by $O\left(N^{4} K^{4} C\right)$, where $C$ is encoded bits of $a_{i k}$.

\section{HARD-TO-MANIPULATE}

In this section, we attempt to break the assumption where bidders are risk-neutral by proposing a hard-to-manipulate auction. Instead of performing a single greedy algorithm, we attempt local improvements in the ordering of the bidders. Specifically, we repeatedly improve the solution quality by making a single change in the order of the bids, and run a greedy algorithm on the modified order. The improvements can improve social welfare and spectrum utilization. However, such an efficient allocation algorithm will not guarantee the monotonicity which makes the payment scheme hard to be designed to ensure truthfulness. We solve the problem by moving the difficulty of manipulation from the mechanism to the manipulators. We adopt a concept of hard-to-manipulate that states the problem of computing a useful non-truthful bid given knowledge of the bids from other agents is NP-hard.

Specifically, we perform climbing-hill $(\mathrm{CH})$ based on ETEX, and the corresponding mechanism are referred as HMA (Hard-to-Manipulate Auction). In the following, we give the allocation rule and pricing scheme accordingly.

\section{A. HMA Allocation Rules}

The allocation algorithm proceeds in two phases: In the first phase we sequentially allocate the channels to bidders according to the sorted linear programming solutions. Actually, this phase is the ETEX-Allocation process. In the second phase, we perform a hill-climbing process based on the sorted bids in the first phase. The two steps of the allocation algorithm can be described as follows, and the detailed algorithm is given in Algorithm 3.

- STEP 1: Run ETEX-Allocation algorithm and record the obtained social welfare $W(\pi)$, where $\pi$ is the sorted bidder set according to sorted set $B^{\prime}$. 
- STEP 2: For $j=2$ to $N$, do

- $\pi^{\prime} \leftarrow$ ( $\pi$ with bidder $j$ moved to first place).

- Sequentially allocate exact channels to bidders according to $\pi^{\prime}$ while satisfying interference constraints. Let $W\left(\pi^{\prime}\right)$ denote the obtained social welfare on $\pi^{\prime}$. If $W\left(\pi^{\prime}\right) \geq W(\pi)$, return the allocation on $\pi^{\prime}$.

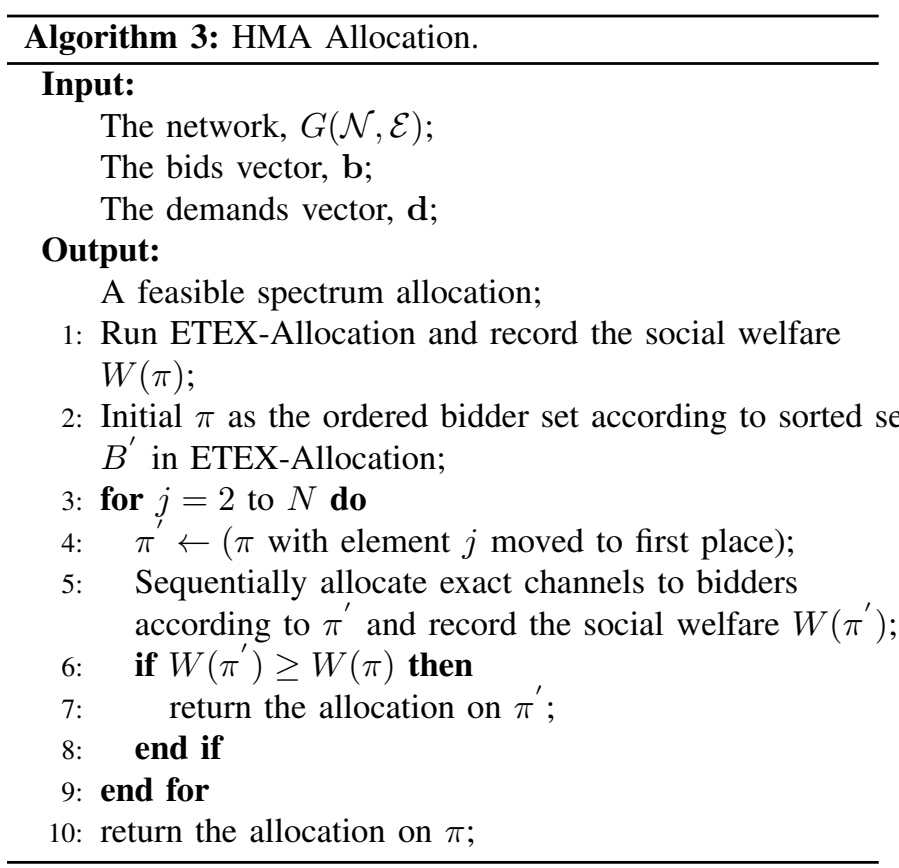

Due to the local improvement is performed, the monotonicity will not be guaranteed. As a result, a threshold payment does not exist for ensuring the truthfulness. We therefore redesign the payment scheme based on the VCG payment scheme.

\section{B. A VCG-Based Payment Scheme}

The idea of the pricing scheme is similar with VCG mechanism. Intuitively, the payment of winner $i$ is the social welfare for everyone else without $i$ in the system minus that for everyone else with $i$ in the system. For easy description, we use $H$ denote the above HMA allocation algorithm, then the price $p_{i}$ we charge from each winner $i$ is:

$$
p_{i}=W_{H}\left(\mathbf{b}_{-i}\right)-\left(W_{H}(\mathbf{b})-b_{i}\right)
$$

Where the item $W_{H}\left(\mathbf{b}_{-i}\right)$ and $W_{H}(\mathbf{b})$ are the social welfare of $H$ running on the bids $\mathbf{b}_{-i}$ and bids $\mathbf{b}$, respectively.

\section{Properties Analysis}

Since our heuristic approach HMA is not optimal and thus the truthfulness will not be guaranteed due to the limitation of VCG mechanism. However, we show manipulating such an auction of a bidder for profit gaining will be hard. We first define the manipulation problem and then prove that it is NPhard.

Definition 3 ( $H$ Manipulation Problem): For the spectrum auction, given bids set $(\mathbf{d}, \mathbf{b})$ where $\mathbf{d}$ is the vector of demands $\left(d_{1}, \cdots, d_{N}\right)$ and $\mathbf{b}$ is the vector of bids $\left(b_{1}, \cdots, b_{N}\right)$, returning a bid $\left(\tilde{d}_{i}, \tilde{b}_{i}\right)$ that gives bidder $i$ at least $\epsilon(\epsilon>0)$ additional utility than what it would have received from being truthful in that auction, or outputting "impossible" if no such manipulation exists.

Definition 4 (Simple-Case H Manipulation Problem):

Consider a simple case for the spectrum auction where conflict graph is complete graph, given bids set $(\mathbf{d}, \mathbf{b})$, returning a bid $\left(\tilde{d}_{i}, \tilde{b}_{i}\right)$ that gives bidder $i$ at least $\epsilon(\epsilon>0)$ additional utility than what it would have received from being truthful in that auction, or outputting "impossible" if no such manipulation exists.

To prove the $H$ manipulation problem is NP-hard, we just need to prove the simple case $H$ Manipulation Problem is NP-hard. A recent work in [25] has investigated the hardto-manipulate auctions and consider the necessary properties of VCG-based auctions that will allow us to say that their manipulation is hard. The main result in [25] is that the allocation algorithm of a VCG-based auction satisfying the properties of Greedy Optimality (called "G-OPT") and Strong Consumer Sovereignty (called "SCS") are the necessary condition on the auction that make manipulation NP-hard. As an instance, the authors show that the condition is also the sufficient condition for the VCG-based combinatorial auction [26], which is a classic economic auction. Inspired by this, we prove the Simple-Case $H$ Manipulation problem is NPHard by showing our allocation algorithm satisfying G-OPT and SCS which are defined in the following.

Definition 5 (Greedy Optimality): An allocation algorithm satisfies greedy optimality if it ensures that the algorithm does not leave channels unassigned when other request them, i.e., there does not exist a losing bidder whose demand $d_{i}$ is less than the available channels, and does not assign more channels than that it requests, i.e., a bidder obtains either exact $d_{i}$ channels or zero channel.

Definition 6 (Strong Consumer Sovereignty): An allocation algorithm satisfies strong consumer sovereignty if there does not exit a single bidder would contribute more social welfare than the total value the algorithm achieves, i.e., there does not exist a bidder $i$ with $b_{i}>W$, where $W$ is the total social welfare achieved by the algorithm.

Based on the definitions, we can prove the following two lemmas.

Lemma 5: The allocation algorithm $H$ satisfies Greedy Optimality.

Proof: Firstly, the allocation algorithm $H$ scans through the bids set, allocates channels to bidders that can be allocated to. At the end, either all bidders are allocated to, or any remaining bidders could not possibly be allocated to given the current allocation. Secondly, since we adopt the strict request here, we allocate the exact $d_{i}$ or zero channels to bidder $i$. This completes the proof.

Lemma 6: The allocation algorithm $H$ satisfies Strong Consumer Sovereignty.

Proof: We assume that there exists a bidder $i$ whose $b_{i}>W$ where $W$ is the total social welfare achieved by the allocation algorithm $H$. If bidder $i$ wins in the allocation, then $W$ must contain the $b_{i}$ which indicates $W \geq b_{i}$ and leads to a contradiction. If $i$ loses in the allocation, then by STEP 2 of $H$, 
bidder $i$ will win the auction and this leads to a contradiction.

Now we provide a simple NP-Hard problem (see Lemma 7), and then we show deducing the Simple-Case $H$ Manipulation Problem to it.

Lemma 7: The following problem (Simple-Case-WinnerDetermination) is NP-Hard: Consider the simple case for the spectrum auction where conflict graph $G$ is complete graph, determining whether an allocation can achieve $V$ total valuation (social welfare), and outputting that allocation if it exists.

Proof: The simple case of spectrum auction with maximizing social welfare can be reduced to classic 0/1 Knapsack Problem, where bid is equivalent to 'value' and demand is equivalent to 'weight'. Therefore the above problem is equivalent to the decision form of classic 0/1 Knapsack Problem, which is known to be NP-hard.

Now we can prove the Simple-Case $H$ Manipulation Problem is NP-Hard by deducing it to the Simple-Case-WinnerDetermination problem.

Theorem 3: The Simple-Case H Manipulation Problem is NP-Hard.

Proof: Suppose the Simple-Case Manipulation Problem could be solved in polynomial time. We provide a polynomial time solution to Simple-Case-Winner-Determination.

Denote $\theta$ as a vector $\left(\left(d_{1}, v_{1}\right), \cdots,\left(d_{N}, v_{N}\right)\right)$ where $N$ is the number of bidders. First, run the allocation algorithm on $\theta$. Let the total valuation of the result be $x$. If $x \geq V$, then we are done: output "yes" and return the allocation, where $V$ is the total valuation. We suppose otherwise, i.e., $V>x$.

Denote $\theta^{\prime}=\left(\left(d_{1}, v_{1}\right), \cdots,\left(d_{N}, v_{N}\right),(K, V-\epsilon)\right)$. We know that $V>x$, and thus $V-\epsilon>x$ (since $\epsilon$ is of arbitrarily small precision). Therefore, when run on $\theta^{\prime}$, the allocation algorithm will allocate total $K$ channels to $N+1$ by SCS. Since no one else can be allocated to, agent $N+1$ 's payment is $x$, and its utility is $V-x-\epsilon$.

Now we prove the main reduction: $\theta$ has an allocation achieving $V$ total valuation if only and if bidder $N+1$ can achieve at least $\epsilon$ units of manipulating in $\theta^{\prime}$. Since the next derivation process is similar with that of VCG-based combinatorial auction in [25], we omit here.

Based on the above understanding, we claim the $H$ Manipulation Problem is NP-Hard.

Theorem 4: The H Manipulation Problem is NP-Hard.

Proof: Since $H$ Manipulation Problem generalizes the Simple-Case $H$ Manipulation Problem, it is only harder.

Computational Complexity: We now analyze the computational complexity of HMA. The complexity of HMA allocation consists of two parts, i.e., STEP 1 and STEP 2. The complexity comes form step 1 is the complexity of ETEX-Allocation, i.e., $O\left(L+N \log N+N^{2}\right)$ plus the time consumed by LP process. The complexity comes from step 2, which needs at most $N^{2}$ times of assigning and updating process. This step needs $O\left(N^{3}\right)$ time. To charge each winner in pricing scheme, we need to run HMA allocation algorithm once again without BFS procedure. Therefore the total complexity of HMA is time $O\left(L+N^{2} \log N+N^{3}+N^{4}\right)$ plus $\sharp+1$ times of the time consumed by the LP process with bounded $K \times N$ parameters, where $\sharp$ is the number of winners in the auction.

\section{PERFormance EVAluation}

In this section, we use simulation experiments to evaluate the performance of the approximately truthful mechanisms.

\section{A. Simulation Methodology}

1) Network Topology. We assume a single auctioneer that conducts an auction in a relatively small geographic area. Bidders are randomly deployed in a square of $1.0 \times$ 1.0 area. We vary the network density by varying the number of bidders from 50 to 500 (the default is 300). To generate the interference graph, we set the interference range as 0.1 , i.e., if the distance between any two bidders is less than 0.1 , they will interfere with each other when using the same channel simultaneously.

2) Channels, Bids, and Demands. We set up an auction of total channels varying from 2 to 20 with the default being 6 channels. The per-channel bid of bidder $i$ (i.e., $\left.b_{i} / d_{i}\right)$ is randomly distributed within $(0,1]$. The channel demand of bidder $i$ (i.e., $\left.d_{i}\right)$ is randomly chosen from the interval $[1, K]$ where $K$ is the total number of available channels.

3) Performance Metrics. We use the following performance metrics to evaluate the efficiency:

- Social Welfare: the sum of all winners' bids, i.e., $\sum_{i=1}^{i=N} x_{i} b_{i}$

- Spectrum Utilization: the sum of allocated channels over winners, i.e., $\sum_{i=1}^{i=N} x_{i} d_{i}$.

- Bidder Satisfaction: the percentage of winners, i.e., $\frac{\sum_{i=1}^{i=N} x_{i}}{N}$.

\section{B. Approximately Truthful vs. Truthful}

We now evaluate the performance on the approximately truthful mechanisms by comparing them with VERITAS [14] and the method in [21] (referred as SW-Fair). VERITAS is one of the most famous SSA with the objective of maximizing social welfare. VERITAS consists of a greedy allocation algorithm and a critical value based pricing method. SW-Fair is also a greedy-based mechanism. Here we describe these mechanisms briefly in the following:

VERITAS-Allocation: Firstly, the per-channel bids of all bidders are sorted from high to low, then the algorithm allocates exact channels to bidders sequentially from the bidder with the highest bid to the lowest one. For each bidder $i$, the algorithm first checks whether there are enough channels to satisfy the request while satisfying interference constraints. If so, the request of bidder $i$ is satisfied, otherwise means the opposite.

VERITAS-Pricing: VERITAS charges each winner $i$ with the per-channel bid of its critical neighbor multiplied by the number of channels allocated to $i$. The critical neighbor of bidder $i$ is the first winning neighbor who makes the number of $i$ 's available channels below its demand $d_{i}$ when running VERITAS-Allocation on bids $\mathbf{b}_{-i}$. 


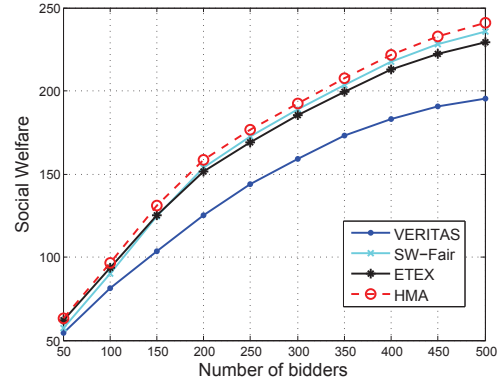

(a)

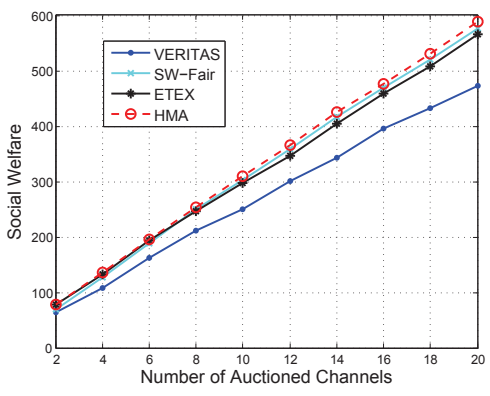

(b)

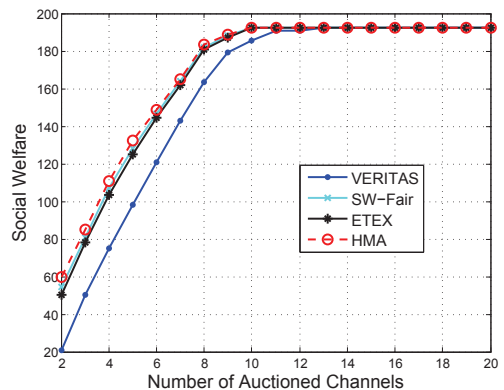

(c)

Fig. 3: Generated social welfare of auction mechanisms. The default number of auctioned channels is 6 (figure (a)), the default number of bidders is 300 (figure (b)) and the channel demand of each bidder is set to 2 (figures (c)).

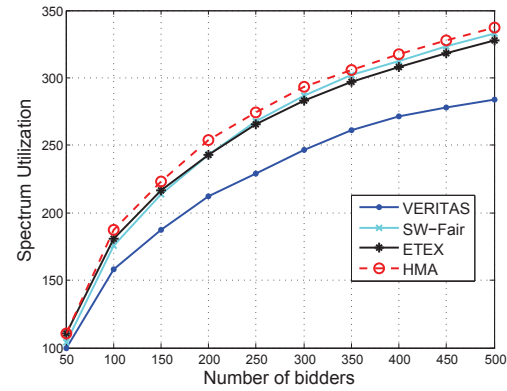

(a)

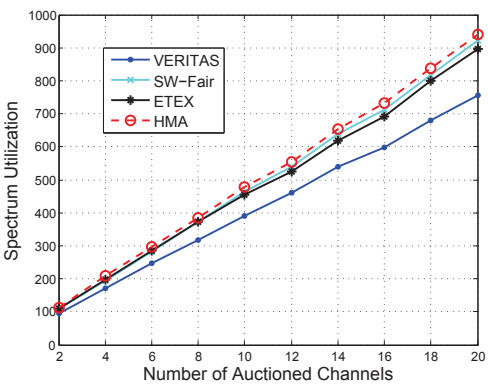

(b)

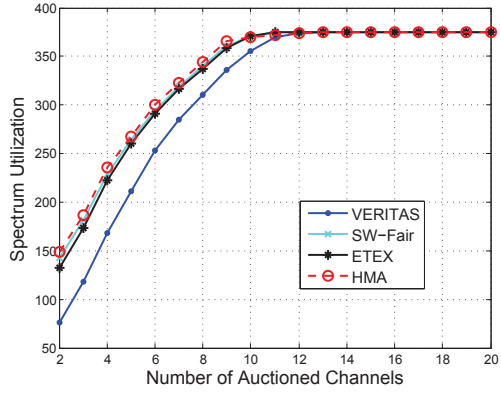

(c)

Fig. 4: Generated spectrum utilization of auction mechanisms. The default number of auctioned channels is 6 (figure (a)), the default number of bidders is 300 (figure (b)) and the channel demand of each bidder is set to 2 (figures (c)).

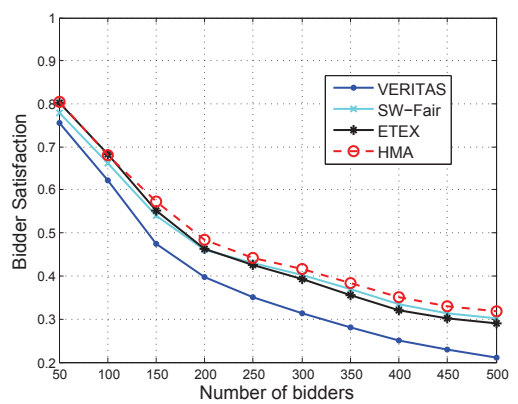

(a)

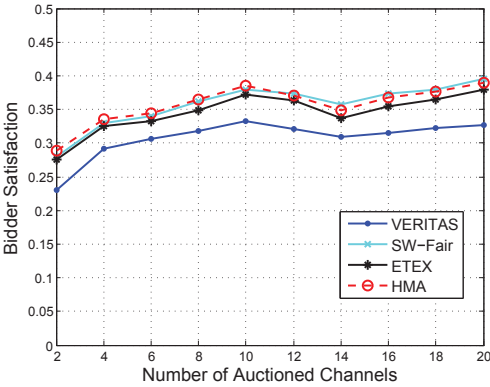

(b)

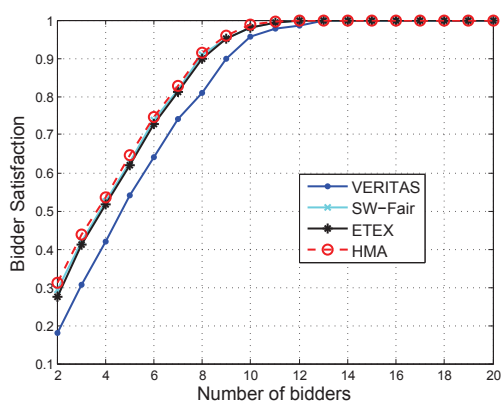

(c)

Fig. 5: Generated bidder satisfaction of the mechanisms. The default number of auctioned channels is 6 (figure (a)), the default number of bidders is 300 (figure (b)), and the channel demand of each bidder is 2 (figure (c)).

SW-Fair is similar with VERITAS, while SW-Fair aims to achieve a trade-off between social welfare and fairness. The main difference between SW-Fair and VERITAS is that SWFair considers the impact to neighbouring nodes when sorting bidders, i.e., SW-Fair sorts the bidders by the computed virtual bid, which is defined as $\frac{b_{i}}{|\mathbb{N}(i)|+1}$ for bidder $i$, where $|\mathbb{N}(i)|$ is the number of $i$ 's neighbors. The detailed algorithm can be referred in [21].

Now we compare VERITAS and SW-Fair with our mechanisms. The key distinction between them is that our mechanisms seek to improve the efficiency in terms of social welfare and spectrum usage. We compare these mechanisms in three scenarios. First, we fix the number of auctioned channels $(K=6)$ and vary the number of bidders from 50 to 500 . Second, we fix the number of bidders $N=300$ and vary the number of auctioned channels from 2 to 20 . In the above two scenarios, the demand of each bidder $d_{i}$ is randomly chosen from the interval $[1, K]$ where $K$ is the total number of available channels. Finally, we fix the demand of each bidder $\left(d_{i}=2\right)$, fix the number of bidders $(N=300)$, and vary the number of auctioned channels from 2 to 20.

We plot the results in Fig. 3, Fig. 4 and Fig. 5. From these results, we observe that: (1) ETEX and HMA outperforms slightly better than VERITAS by about $23 \%$ and $27 \%$ in 


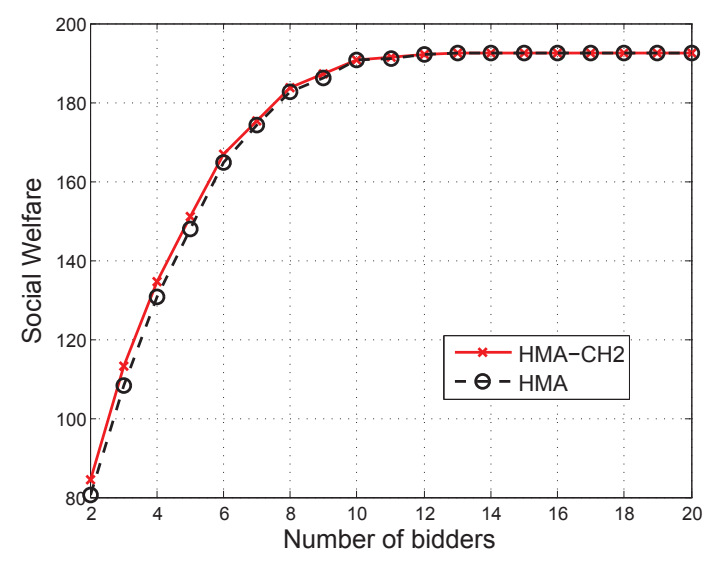

Fig. 6: Evaluate the performance on the further improvements on hill climbing, where HMA-CH2 is the two element improvement on ETEX.

average respectively. (2) The hill-climbing increases the efficiency (social welfare and spectrum utilization) by about $4 \%$ in average. (3) Our mechanisms performs slightly better than SW-Fair, but when the number of bidders increases, SW-Fair performs better than ETEX while is slightly worse than HMA (See Fig. 3(a) and Fig. 4(a)). This is because that the impact of neighboring nodes when allocation is enlarged when the number of bidders increases (i.e., more bidders would conflict). (4) In the first two scenarios, the performance gap increases with the growing in the number of bidders (channels). The main reason for the increased performance of our methods is due to the higher bidder satisfaction which we plot in Fig. 5. That is more bidders succeed in our mechanisms. The performance gap increases as the number of bidders (auctioned channels ) grows confirmed the intuition that the LP-based allocation methods performs well since LP takes mutual interference between neighbours into consideration when allocation. This intuition is also confirmed in [31]. In the third scenario, the performance gap decreases with the growing in the number of channels. This is because more bidders can be satisfied and they can be all allocated with channels when the number of channels is large enough. (5) The metric of bidder satisfaction reflects the fairness of these mechanisms when we use the Jain's fairness index [34] to quantify the fairness of our mechanisms. The Jain's fairness index is computed as $\frac{1}{N} \sum_{i=1}^{N} \frac{X_{i}}{X_{f}}$, where $X_{i}$ denotes the allocation of bidder $i$ and $X_{f}=\frac{\sum_{i=1}^{N} X_{i}^{2}}{\sum_{i=1}^{N} X_{i}}$ is the fair allocation mark. Because we adopt strict request model in our context, i.e., $X_{i}$ is a binary variable which indicates whether $i$ wins or loses. As a result, the Jain's fairness index here means the bidder satisfaction. Therefore, we claim our mechanisms performs better than other methods in terms of fairness by the results in Fig. 5 .

\section{Further Improvements on Hill Climbing}

Recall that the local improvements method in hard-tomanipulate auction, where we just simply move the element $j$

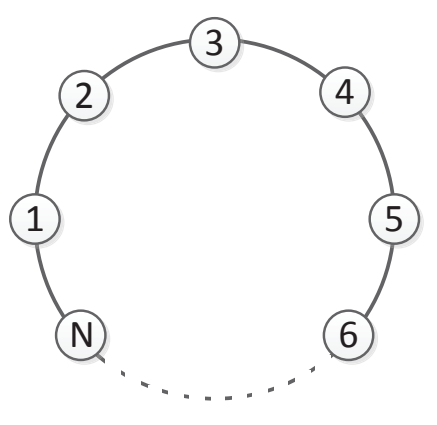

(a) Ring Topology

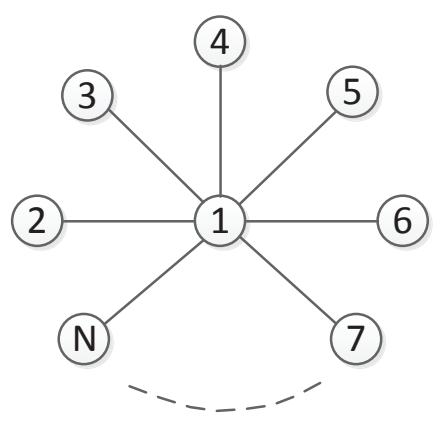

(b) Star Topology
Fig. 7: Fixed Topolgies: Ring (left) and Star (right).

TABLE I: Comparison to Optimum on Random Topologies

\begin{tabular}{|c|c|c|}
\hline Performance Loss & Social Welfare (\%) & Spectrum Utilization (\%) \\
\hline VERITAS & 5.24 & 8.15 \\
\hline SW-Fair & 3.02 & 4.68 \\
\hline ETEX & 3.16 & 5.02 \\
\hline HMA & 2.62 & 3.88 \\
\hline
\end{tabular}

$(j=1, \cdots, N)$ to the first place. Here we tried a further improvement where two elements $(i=1, \cdots, N-1$ and $j=i+1, \cdots, N)$ are moved to the first and second position in the order accordingly. We referred this modified mechanism as HMA-CH2. We compared the performance of the two variants of improvements. The results are given in Fig. 6. From the results, we observe that the performance gap is no more than $2 \%$, while the two element variation costs more computational complexity than our simple improvement.

\section{Compare to the Optimal Solution}

In this section, we now compare the performance derived from our proposed heuristic approaches to the optimal value. Given the complexity of the exhaustive search scales exponentially with the number of bidders, we use two typical topologies (see Fig. 7) with limited number of bidders and channels. Topology I and II are two extreme topologies: a star topology with one vertex interferes with the rest and a ring topology with uniformed interference condition.

Fig. 8 and Fig.9 summarize the results for 20 nodes ring topology and 20 nodes star topology, assuming 2 channels are auctioned. Results are represented as mean of 10 runs. We observe that our proposed approaches achieve similar performance compared to the global optimal.

Following that, we also consider a set of small random topologies assuming 2 channels to be auctioned to 20 bidders. For a clear illustration, we evaluate the performance by computing the performance loss compared to the optimum. If the obtained result is $T$ and the global optimum is $T_{o p t}$, the performance loss is defined as $1-T / T_{\text {opt }}$. This definition is natural and can measure the difference of performance provided by our mechanisms and the global optimum. We summarized the results averaged over 100 random topologies 


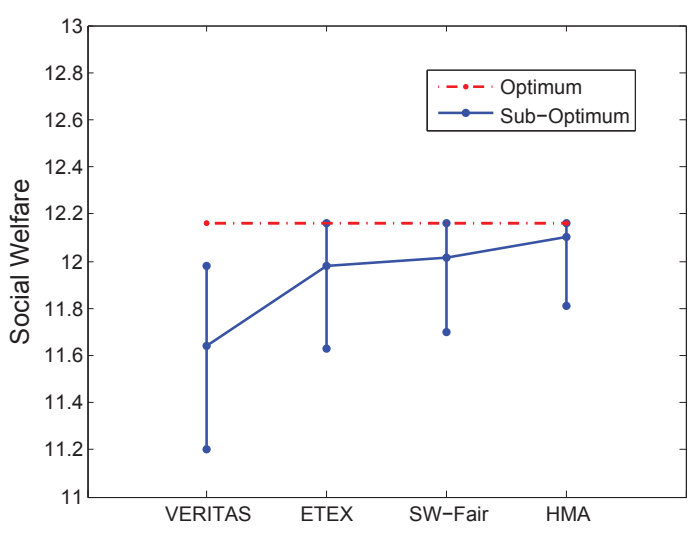

(a) Social Welfare

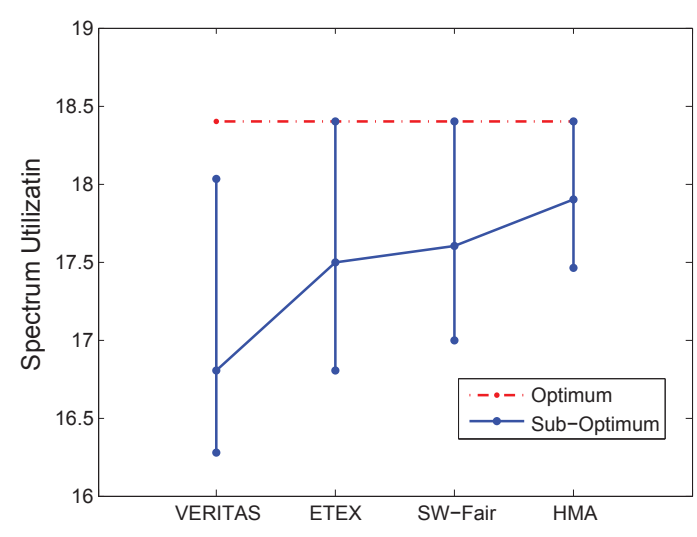

(b) Spectrum Utilization

Fig. 8: Comparison to the optimal solution using 20 nodes ring topology. The channel request of each bidder is 2 .

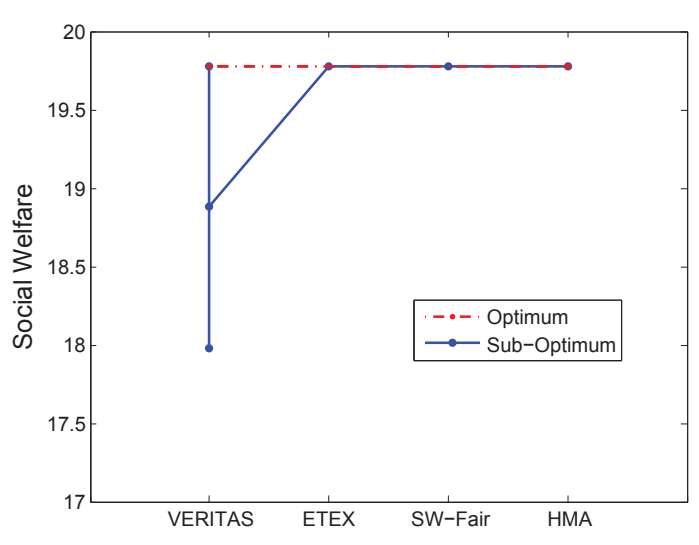

(a) Social Welfare

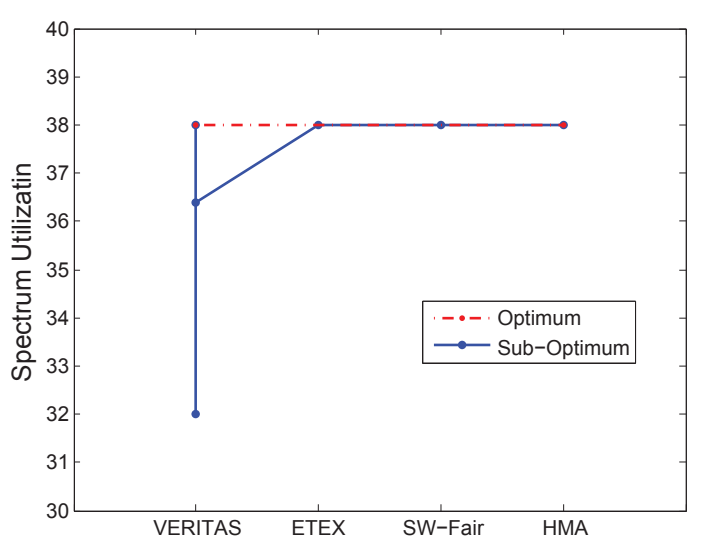

(b) Spectrum Utilization

Fig. 9: Comparison to the optimal solution using 20 nodes star topology. The channel request of each bidder is 2 .

in Table I. We observe that our mechanism HMA provides the better approximation compared to other methods again.

Overall, our methods provide better approximation compared to VERITAS. This is because our methods are based on LP solutions which take mutual influence between neighboring bidders into consideration, and thus achieve better approximation.

\section{CONCLUSION}

In this paper, we study the secondary spectrum auction with single seller and multiple buyers (bidders). We release the burden of ensuring the hard truthfulness by introducing the concept of approximate truthfulness. We show that finding the optimal spectrum allocation under strict-request model is NPhard. To solve this problem, we first provide a suboptimal algorithm which is shown to be monotone. To minimize the computational overhead, we propose a novel pricing scheme to ensure the mechanism containing the monotone algorithm along with the pricing scheme achieving truthful in expectation. Following that, we perform hill-climbing based on the monotone algorithm, which improves the social welfare and spectrum utilization but breaks the monotonicity. Although the novel pricing scheme is no longer suitable, we show it is a hard-to-manipulate auction when combining with a VCGbased payment scheme. Compared to the mechanism achieving truthful in expectation, the hard-to-manipulate auction breaks the assumption that bidders are risk-neutral and improves the allocating efficiency. Theoretical analysis shows the computational efficiency of these approximately truthful mechanisms, and simulation results show that our approximately truthful mechanisms can achieve significant improvement over the state-of-the-art mechanisms.

\section{REFERENCES}

[1] FCC Spectrum Policy Task Force, "Report of the Spectrum Efficiency Working Group", Available: http://www.fcc.gov/sptf/reports.html, 2002.

[2] M.A. McHenry, P.A. Tenhula, D. McCloskey, D.A. Roberson, and C.S. Hood, "Chicago spectrum occupancy measurements \& analysis and a long-term studies proposal", In Proc. of Int. Workshop on Technology and Policy for Accessing Spectrum, ACM New York, USA, 2006.

[3] I. Akyildiz, W. Y. Lee, M. Vuran, and S. Mohanty, "NeXt generation/dynamic spectrum access/cognitive radio wireless networks: a survey", In Computer Networks, 50(13), pp. 2127-2159, 2006.

[4] Robert J. Berger, "Open Spectrum: A Path to Ubiquitous Connectivity", In ACM QUEUE, 1(3), pp.60-68, 2003. 
[5] P. Klemperer, "Auctions, theory and practice", Princeton University Press, USA, 2004

[6] P. Gupta and P. R. Kumar, "The capacity of wireless networks," IEEE Transactions on Information Theory, 46(2), pp.388-404, 2000.

[7] K. Jain, J. Padhye, V. N. Padmanabhan, and L. Qiu, "Impact of interference on multi-hop wireless network performance", In Proc. of ACM MOBICOM, pp. 66-80, 2003.

[8] Q. Wang, B. Ye, T. Xu, and S. Lu, "An Approximate Truthfulness Motivated Spectrum Auction for Dynamic Spectrum Access", In Proc. of IEEE WCNC, pp.257-262, 2011.

[9] N. Nisan and A. Ronen, "Computationally feasible VCG mechanism", Journal of Artificial Intelligence Research, vol. 29, pp. 19-47, 2007.

[10] A. Kothari, D. C. Parke, and S. Suri, "Approximately strategyproof and tractable multi-unit auctions", In Proc. of ACM Electronic Commerce, pp. 166-175, 2003.

[11] Y. Wu, B. Wang, K. Liu, and T. Clancy, "A scalable collusion resistant multi-winner cognitive spectrum auction game", IEEE Trans.on Communications, vol. 57, pp. 3805-3816, 2009.

[12] S. Gandhi, C. Buragohain, L. Cao, H. Zheng, and S. Suri, "A General Framework for Wireless Spectrum Auction", In Proc. of IEEE DySPAN, pp. 22-33, 2007.

[13] A.P. Subramanian, M. Al-Al-Ayyoub, H. Gupta, S.R. Das, and M.M. Buddhikot, "Near-Optimal Dynamic Spectrum Allocation in Cellular Networks", In Proc. of IEEE DySPAN, pp.1-11, 2008.

[14] X. Zhou, S. Gandhi, S. Suri, and H. Zheng, "eBay in the Sky: StrategyProof Wireless Spectrum Auctions", In Proc. of ACM MOBICOM, pp.213, 2008

[15] J. Jia, Q. Zhang, Q. Zhang, and M. Liu, "Revenue Generation for Truthful Spectrum Auction in Dynamic Spectrum Access", In Proc. of ACM MOBIHOC, pp. 3-12, 2009.

[16] X. Zhou, H. Zheng, "TRUST: A General Framework for Truthful Double Spectrum Auctions", In Proc. of IEEE INFOCOM, pp.999-1007, 2009.

[17] T. Groves, "Incentives in teams", Econometrica: Journal of the Econometric Society, pp. 617-631, 1973.

[18] W. Vickrey, "Counter speculation, auctions, and competitive sealed tenders", Journal of Finance, pp. 8-37, 1961.

[19] E. Clarke, "Multipart pricing of public goods", Public choice, vol. 11, pp. 17-33, 1971.

[20] M. Al-Ayyoub, H. Gupta, "Truthful spectrum auctions with approximate revenue", In Proc. of IEEE INFOCOM, pp.2813-2821, 2011.

[21] Z. Li, C.Wu, "Strategyproof Auctions for Balancing Social Welfare and Fairness in Secondary Spectrum Markets," In Proc. of IEEE INFOCOM, pp.3020-3028, 2011.

[22] A. Archer, C. H. Papadimitriou, K. Talwar, and Eva Tardos, "An approximate truthful mechanism for combinatorial auctions with single parameter agents", Internet Mathematics, 1(2), pp.129-150, 2004.

[23] H. Karloff, "Linear programming", Birkhauser, 1991.

[24] A. Mu'alem, N. Nisan, "Truthful Approximate Mechanisms for Restricted Combinatorial Auctions", In Proc. of AAAI, pp.379-384, 2002.

[25] S. Sanghvi, D. C. Parkes, "Hard-to-Manipulate VCG-Based Auctions", Harvard University Technical Report, 2004

[26] S. de Vries, R. Vohra, "Combinatorial auctions: A survey", 2000

[27] N. Nisan, T. Roughgarden, Eva Tardos and Vijay V. Vazirani, "Algorithmic Game Theory", Cambridge University Press, 2007.

[28] M. H. Rothkopf, A. Pekec, and R. M. Harstad, "Computationally manageable combinatorial auctions", Management Science, pp.1131-1147, 1998.

[29] T. Sandholm, "Algorithm for optimal winner determination in combinatorial auctions", Artificial Intelligence, pp.1-54, 2002.

[30] J. Feigenbaum and S. Shenker, "Distributed Algorithmic Mechanism Design: Recent Results and Future Directions", In Proc. of International Workshop on Discrete Algorithms and Methods for Mobile Computing and Communications, pp.1-13, 2002.

[31] C. Buragohain, S. Suri, C. Toth, and Y. Zhou, "Improved throughput bounds for interference-aware routing in wireless networks", In UCSB Technical Report, 2006.

[32] Y. Wu et al., "A multi-winner cognitive spectrum auction framework with collusion-resistant mechanisms", In Proc. of DySPAN, pp. 1-9, 2008

[33] Matthew O. Jackson, "Mechanism theory", in The Encyclopedia of Life Support Systems, EOLSS Publishers, 2000.

[34] R. Jain, D. Chiu, and W. Hawe. "A quantitative measure of fairness and discrimination for resource allocation in shared computer systems", $D E C$ Research Report TR-301, 1984.

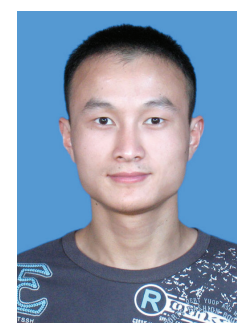

Qinhui Wang received his B.Sc. degree in computer science from Nanjing University, China in 2007. $\mathrm{He}$ is with Department of Military Management, Army Command College, China. He is currently a $\mathrm{PhD}$ candidate at the department of Computer Science and Technology, Nanjing University, China. His current research interests include Cognitive Radio Networks, Spectrum Management and Mobile Wireless Networks.

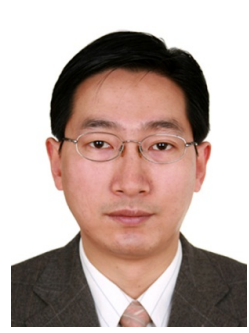

Baoliu Ye received his $\mathrm{PhD}$ degree in computer science from Nanjing University, China in 2004. $\mathrm{He}$ is now an associate professor at the department of Computer Science and Technology, Nanjing University, China. He served as a visiting researcher of the University of Aizu, Japan from March 2005 to July 2006. His current research interests include Peer-to-Peer (P2P) computing, online/mobile social networking, and wireless network. He has published over 40 technical papers in the above areas. $\mathrm{He}$ served as the TPC co-chair of HotPOST'12, HotPOST'11, P2PNet' 10. He is the regent of CCF and a member of IEEE, ACM.

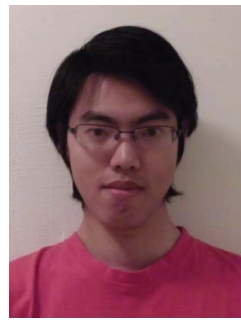

Tianyin Xu is a Ph.D. student in Department of Computer Science and Engineering, University of California San Diego (UCSD). Before joining UCS$\mathrm{D}$, he worked as a research staff in Institute of Computer Science, University of Goettingen, Germany. He received his B.Sc. and M.Eng. Degrees in Computer Science from Nanjing University, China in 2007 and 2010, respectively. His research interests lie broadly in computer systems and networks, with the goal of making todays systems more manageable, reliable, and available.

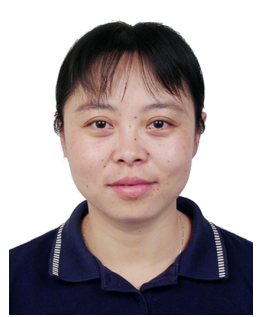

Sanglu Lu received her B.S., M.S., and Ph.D degrees from Nanjing University in 1992, 1995, and 1997 , respectively, all in computer science. She is currently a professor in the Department of Computer Science \& Technology and the State Key Laboratory for Novel Software Technology. Her research interests include distributed computing, wireless networks and pervasive computing. She has published over 80 papers in referred journals and conferences in the above areas. She is a member of IEEE.

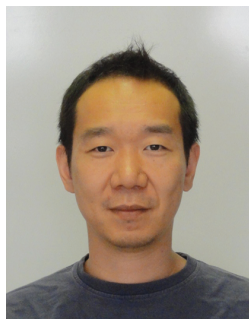

Song Guo (M'02-SM'11) received the $\mathrm{PhD}$ degree in computer science from the University of Ottawa, Canada. He is currently a full professor at School of Computer Science and Engineering, the University of Aizu, Japan. His research interests are mainly in the areas of protocol design and performance analysis for computer and telecommunication networks. He received the Best Paper Awards at ACM IMCOM 2014, IEEE CSE 2011, and IEEE HPCC 2008. Dr. Guo currently serves as an Associate Editor of the IEEE Transactions on Parallel and Distributed Systems. He is in the editorial boards of ACM/Springer Wireless Networks, Wireless Communications and Mobile Computing, and many others. He has also been in organizing and technical committees of numerous international conferences, including serving as a General Chair of MobiQuitous 2013. Dr. Guo is a senior member of the IEEE and the ACM. 